\section{microRNA-24a is required to repress apoptosis in the developing neural retina}

\author{
James C. Walker and Richard M. Harland ${ }^{1}$ \\ Department of Molecular and Cell Biology and Center for \\ Integrative Genomics, University of California at Berkeley, \\ Berkeley, California 94720, USA
}

Programmed cell death is important for the proper development of the retina, and microRNAs (miRNAs) may be critical for its regulation. Here, we report that miR$24 a$ is expressed in the neural retina and is required for correct eye morphogenesis in Xenopus. Inhibition of miR-24a during development causes a reduction in eye size due to a significant increase in apoptosis in the retina, whereas overexpression of $\mathrm{miR}-24 a$ is sufficient to prevent apoptosis. We show that miR-24a negatively regulates the proapoptotic factors caspase9 and apaf1, demonstrating a role for miRNAs in the regulation of apoptosis during normal development.

Supplemental material is available at http://www.genesdev.org.

Received January 3, 2009; revised version accepted March 24, 2009.

Programmed cell death, or apoptosis, regulates the size and morphology of developing tissues and organs, and this is particularly true in the developing neural retina. The neural retina gives rise to the photoreceptors, retinal ganglion cells, and the optic nerve, and as many as $90 \%$ of newborn retinal ganglion cells die during rat retinal development (de la Rosa and de Pablo 2000; Vecino et al. 2004). In Xenopus, spatiotemporal elimination of retinal cells is a key factor in maturation (Gaze and Grant 1992). In the chick, Caspase-dependent apoptosis has been demonstrated in the retina, and inhibition of Caspases results in an enlargement of the ganglion cell layer (Mayordomo et al. 2003). Similarly, in mice, apoptotic factors are highly expressed in the early retina and downregulated as development proceeds (O'Driscoll et al. 2006; Wallace et al. 2006), and knockdown of Caspases results in an overgrown retina (Hakem et al. 1998). However, the factors important for the regulation of Caspases and other apoptotic factors in the eye are unknown, although some transcriptional regulation has been postulated (Wallace et al. 2006).

microRNAs (miRNAs), a class of regulatory noncoding RNA genes, have evolutionarily conserved roles during the development of many organs, including the heart and nervous system (Zhao et al. 2005; Makeyev et al. 2007). miRNAs are transcribed by RNA Polymerase II as pri-

[Keywords: microRNAs; $\mathrm{miR}-24 a$; apoptosis; caspase9; apaf1] ${ }^{1}$ Corresponding author.

E-MAIL Harland@berkeley.edu; FAX (510) 643-1729.

Article published online ahead of print. Article and publication date are online at http://www.genesdev.org/cgi/doi/10.1101/gad.1777709. mary miRNAs (pri-miRNAs). These transcripts are then processed by the enzymes Drosha and Dicer to generate the mature single-stranded miRNA of $\sim 22$ nucleotides, which is then incorporated into the RNA-induced silencing complex (RISC), characterized by the presence of the Argonaute family of proteins (Pasquinelli et al. 2005). This complex is responsible for the regulatory function of the miRNAs, leading to translational repression or degradation of target mRNAs.

Several miRNAs have been implicated in the regulation of apoptosis in Drosophila (Xu et al. 2004). In various forms of cancer, miR-21 has been shown to be an antiapoptotic factor (Chan et al. 2005; Cheng et al. 2005) and miR-34 has been shown to be a downstream target of p53 and an inducer of cell death (He et al. 2007). However, knowledge is still lacking about the in vivo roles of most miRNAs during vertebrate development. Recent work in mice has shown that Dicer inactivation, specifically in the retina, results in neuronal degeneration (Damiani et al. 2008). Therefore, miRNAs may be important for the regulation of cell death pathways in the retina during development.

In this study, we show through loss-of-function experiments that $m i R-24 a$ is necessary for proper neural retina development. We further demonstrate that knockdown of $m i R-24 a$ results in an increase in apoptosis, while proliferation, patterning, and differentiation of the eye remain unchanged. We find that loss of $m i R-24 a$ function leads to an increase in Caspase9 protein levels without altering mRNA levels, and that the miRNA knockdown phenotype is dependent on the function of Caspase9. In addition, we establish that $m i R-24 a$ is capable of repressing the proapoptotic factors apaf1 and caspase 9 by interaction with their $3^{\prime}$ untranslated regions (UTRs). Finally, we show that $m i R-24 a$ is able to prevent Caspasedependent apoptosis when overexpressed. Together these data indicate that $m i R-24 a$ is an essential regulator of caspase 9 and programmed cell death in the developing neural retina.

\section{Results and Discussion}

$\mathrm{miR}-24 \mathrm{a}$ is expressed in the developing neural retina

Previously, we had reported the pri-miRNA expression patterns for several miRNAs during development of Xenopus tropicalis (Walker and Harland 2008). Particularly intriguing was expression of $m i R-24 a$, which appeared in the eye anlage and the posterior mesenchyme during late neurula stages (Fig. 1A). In Xenopus laevis, neural retina development begins when the eye anlagen evaginate from the anterior portion of the neural tube and continues as the optic cup invaginates and later differentiates into three nuclear layers (Nieuwkoop and Faber 1994). Further analysis of the expression of $m i R-24 a$ revealed that this miRNA is expressed specifically in the neural retina throughout the development of the eye until at least stage 40 (Fig. 1B-D'; data not shown). Results obtained by in situ hybridization were confirmed by RT-PCR (Fig. 1E).

\section{Knockdown of miR-24a results in reduced eye size}

To determine the function of $m i R-24 a$ during development, we used an antisense morpholino oligonucleotide 

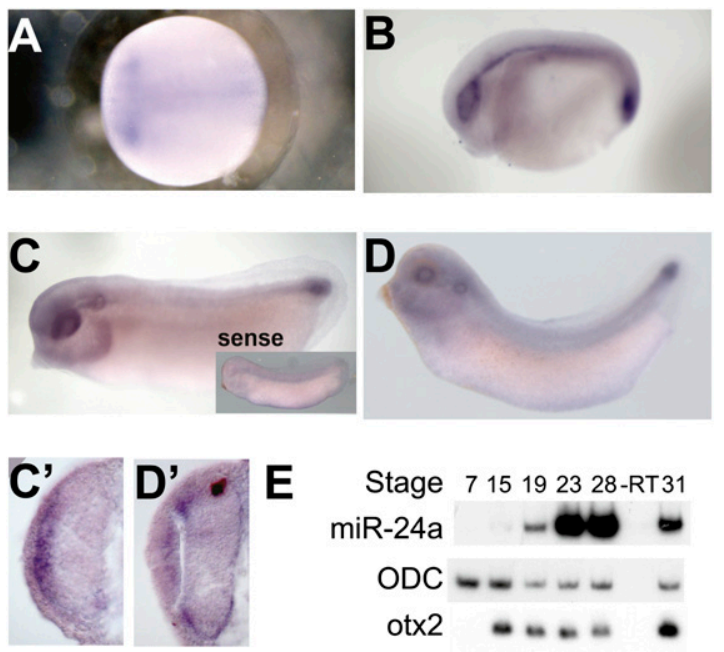

Figure 1. $m i R-24 a$ is expressed in the developing neural retina. In situ hybridization shows expression of pri-miR-24a in the embryonic eye anlagen and posterior mesenchyme (stage $19[A]$ and stage $23[B]$. Expression continues in the neural retina as the optic cup develops and invaginates (stage $28[C]$ and stage $31[D])$. (C, inset) Sense probe shows no expression. (E) RT-PCR for the primary miRNA from stages 7 to 31 shows that miR-24a expression begins at stage 19 and is highly expressed throughout maturation.

designed to complement both the mature miR-24a sequence and some sequence of the adjacent loop in the predicted pri-miRNA structure (24aMO). This design was predicted to prevent processing of the primary miRNA transcript as well as to inhibit the function of the mature miRNA (Yin et al. 2008). Injection of $20 \mathrm{ng}$ of $24 \mathrm{aMO}$ in one cell at the two-cell stage resulted in a reduction in eye size (Fig. 2A-F'), which was significant after stage $28(n=$ 94 sections). Eyes containing $24 \mathrm{aMO}$ were reduced $50 \%$ in size compared with the uninjected control eye at stage 40 (data not shown). To ensure the specificity of the morpholino, we performed several control experiments. We were able to rescue the morpholino by coinjecting 2 ng of duplex miR-24a RNA, but not by coinjecting miR-133b RNA (Fig. 2G), showing that the rescue is not due to general miRNA activity. In addition, a mismatch morpholino with four base substitutions in the "seed" region (24ammMO) did not have a phenotype at an equivalent dose (Fig. 2G).

To further ensure that $24 \mathrm{aMO}$ was blocking miR-24a function, we constructed several GFP reporters, where the coding region of a destabilized GFP was fused to a 3'UTR containing miRNA recognition elements (MREs) (see the Supplemental Material). If the miRNA functionally interacts with the recognition element, then the miRNA would repress translation of the GFP and the embryo would lack green fluorescence. RNA coding for RFP without MREs was always coinjected, so that a measure of the repression of GFP by the miRNA could be generated by quantification of the green:red fluorescence ratio. A reporter GFP with two perfect miR-24a recognition elements in its 3'UTR (24aMRE), when coinjected with $500 \mathrm{pg}$ of duplex miR-24a, was translationally repressed (Fig. 2H). However, when $10 \mathrm{ng}$ of $24 \mathrm{aMO}$ were also coinjected, it was able to block the miRNA-induced repression (Fig. $2 \mathrm{H}$ ). This repression was dependent on the miR-24a recognition elements, because mutating these elements (mut24aMRE) resulted in no repression by the miRNA (Fig. 2H). Together, these results show that $24 \mathrm{aMO}$ is able to block the function of miR-24a in vivo, and that this leads to a reduction in eye size.
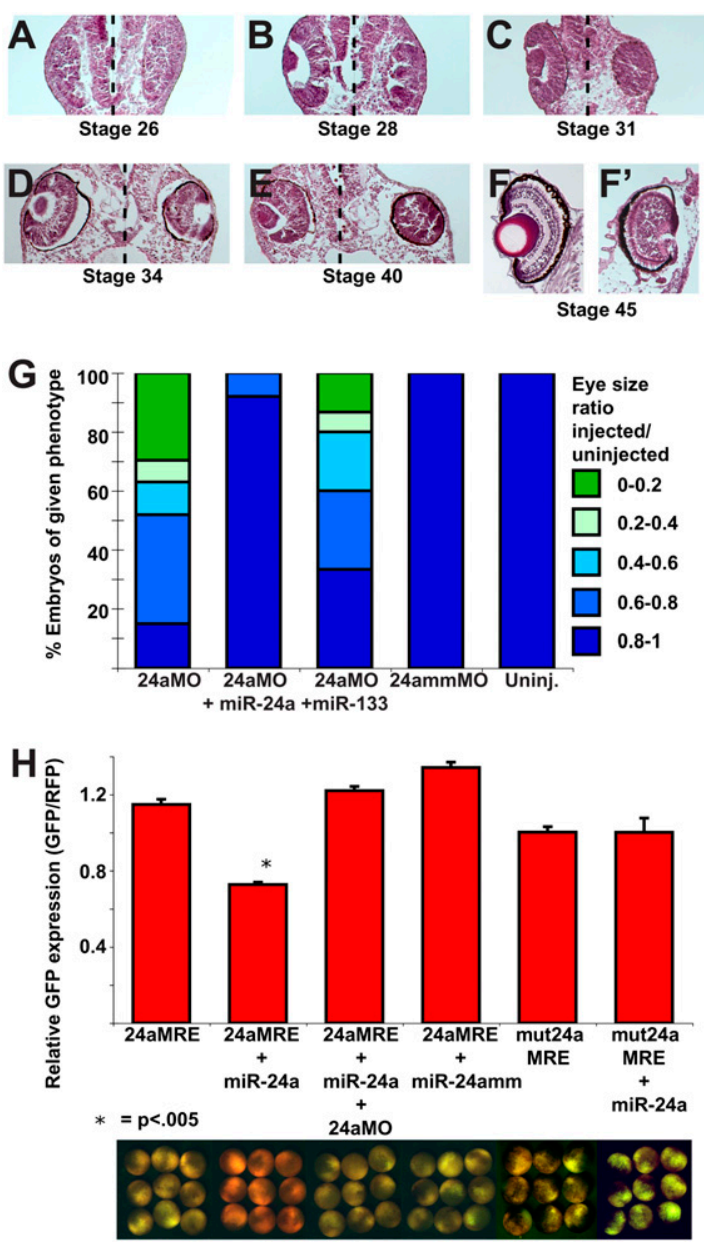

Figure 2. $m i R-24 a$ knockdown results in a reduction in eye size. $\left(A-F^{\prime}\right)$ Hematoxylin and eosin staining of $12-\mu \mathrm{m}$ sections in embryos injected with $20 \mathrm{ng}$ of $24 \mathrm{aMO}$ in one cell at the two-cell stage, injected half on the right. The knockdown of miR-24a causes a reduction in eye size that does not occur until after stage $26(A)$, first becoming prevalent at stage $28(B)$. The reduction in eye size becomes obvious in stage $31(C)$, stage $34(D)$, stage $40(E)$, and stage $45\left(F, F^{\prime}\right)$ embryos (see also Supplemental Fig. 1). $(G)$ The size of the eye was measured at stage 40 and the ratio of the injected eye to the uninjected eye in each embryo was used to classify the severity of phenotypes. miR-24a knockdown caused many embryos to have a smaller eye, an effect that was rescuable by coinjection of $m i R-24 a$ duplex RNA, but not of miR-133b duplex RNA. A mismatch morpholino also had no effect on eye size ratio $(n=121)$. $(H)$ $24 a M O$ functionally represses miR-24a. A GFP construct with two miR-24a recognition elements (24aMRE) when injected alone strongly fluoresces, but shows significantly lower levels of fluorescence when coinjected with duplex miR-24a RNA ( $n=9$; error bars are the standard error of the mean [SEM]). This effect is dependent on the interaction between $m i R-24 a$ and the miR-24a recognition elements, because mutating either abrogates the effect. 24aMO is able to rescue fluorescence of 24aMRE by blocking the function of miR-24a when coinjected. Similar results were obtained with multiple experiments. RFP without miR-24a recognition elements was always coinjected, so that a measure of GFP repression was expressed as the relative fluorescence of GFP/RFP. 


\section{Knockdown of miR-24a leads to an increase in apoptosis}

We first analyzed the gene expression patterns of patterning and differentiation genes in the eye, as well as terminal differentiation markers. There was no change in the levels of expression for any of these genes, indicating that patterning and differentiation were unperturbed (Supplemental Fig. S1). Instead, eye size may be affected by a decrease in proliferation or an increase in apoptosis. Analysis of phosphorylated histone-3, a marker of dividing cells (Saka and Smith 2001), showed no significant difference between the $24 \mathrm{aMO}$-injected side and the uninjected side in the number of proliferating cells in the eye (Fig. 3A). In contrast, TUNEL staining on 24aMO-injected embryos clearly demonstrated that apoptosis was significantly increased in the eye of $m i R-24 a$ knockdown embryos (Fig. 3B). Injection with the mismatch morpholino (24ammMO) had no such increase (data not shown). Furthermore, the increase in apoptosis due to $\mathrm{miR}-24 \mathrm{a}$ knockdown began even before a morphological phenotype was observed, as early as stage 24 , and continued through the development of the eye to tadpole stages (Fig. 3B; data not shown). These results indicate that $m i R-24 a$ may be a potent negative regulator of members of the apoptotic pathway during eye development.
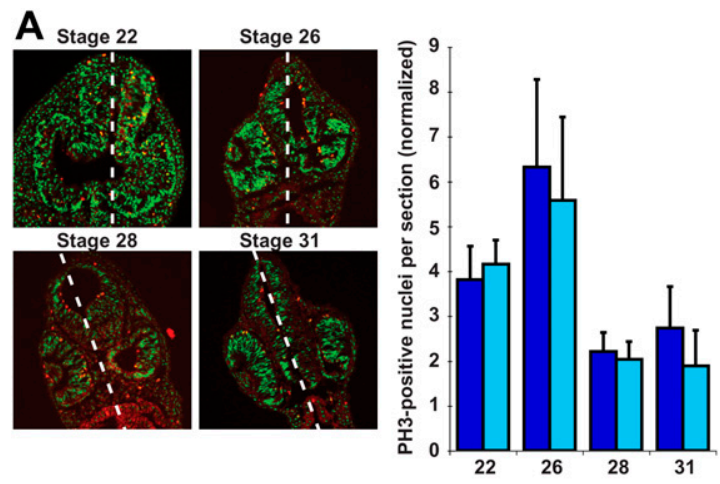

B
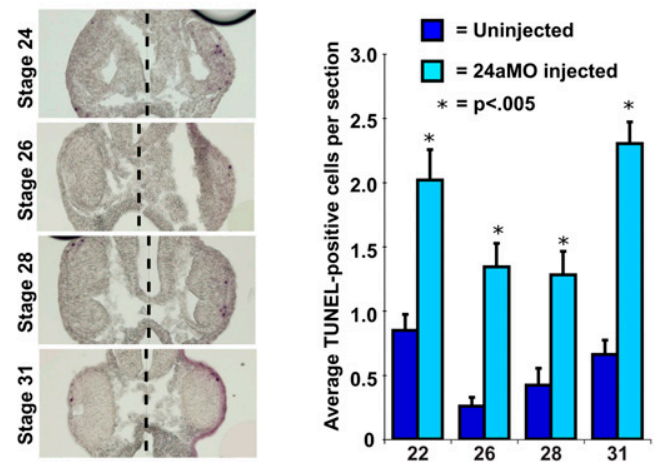

Figure 3. $m i R-24 a$ knockdown has no effect on proliferation, but leads to an increase in apoptosis. (A) Immunohistochemistry for phosphorylated histone3 ( $\mathrm{PH} 3$, in red) shows that there is no significant difference in proliferation between control eyes and 24aMOinjected eyes $(n=6$ embryos per stage; 18 sections per embryo; sections are $12 \mu \mathrm{m}$; error bars are SEM). (B) Knockdown of $m i R-24 a$ results in a significant increase in apoptosis at all stages assayed, measured by the number of TUNEL-positive nuclei per 20 ( $n=6$ embryos per stage; 11 sections per embryo; error bars are SEM).
Knockdown of miR-24a causes up-regulation of Caspase9 protein and inhibition of Caspase9 rescues the knockdown phenotype

One of the most important members of the apoptotic pathway during neural development is caspase9 (Cecconi et al. 2008). Therefore, we examined both caspases mRNA levels by RT-PCR and protein levels by Western blot. In $m i R-24 a$ knockdown embryos, the levels of caspase 9 mRNA were the same as uninjected controls or embryos injected with the mismatch morpholino 24ammMO (Fig. 4A). However, Caspase9 protein levels were increased significantly in embryos with reduced $m i R-24 a$ function, relative to controls (Fig. 4B). These data demonstrate that caspase 9 is regulated posttranscriptionally and may be a direct target of $m i R-24 a$.

To test whether Caspase9 function was necessary for the $m i R-24 a$ knockdown phenotype, we coinjected several specific Caspase inhibitors with 24aMO and analyzed their effect on eye size. Significantly, the only specific inhibitor we tested that was able to fully rescue the 24aMO phenotype was the Caspase9-specific inhibitor Ac-LEHD-CMK (Fig. 4C). An inhibitor to all Caspases was also able to rescue the miR-24a knockdown phenotype (Fig. 4C). Together, these data suggest that $m i R-24 a$ regulates apoptosis by repressing protein translation of caspase 9 mRNA.

\section{miR-24a can negatively regulate the proapoptotic factors caspase9 and apaf1}

We investigated the possibility that members of the apoptotic pathway were direct targets of $m i R-24 a$. First, we analyzed the $3^{\prime}$ UTRs of proapoptotic genes for putative $m i R-24 a$-binding sites from both $X$. tropicalis and $X$. laevis using miRbase (Griffiths-Jones et al. 2008) as well as RNAhybrid analysis of EST databases (Kruger and Rehmsmeier 2006). Both caspase9 and apoptosis protease-activating factor 1 (apaf1) were identified by this method as potential miR-24a targets (Fig. 5A). Caspase9 and Apaf1, along with Cytochrome $\mathrm{C}$ from the mitochondria, form a complex in the cytosol known as the apoptosome, which initiates a catalytic cascade that leads eventually to cell death (Penaloza et al. 2008). Caspase9 and apaf1 are both expressed in the neural retina during the time of development when miR-24a functions (Supplemental Fig. S2).

To test whether $m i R-24 a$ is able to regulate caspase9 and apaf1 directly, we made reporter constructs with the $X$. laevis 3'UTR of these genes fused to a destabilized GFP. In addition, we mutated two putative $m i R-24 a$ recognition elements in the 3'UTRs as controls (mutCasp9 and mutApaf1). $m i R-24 a$ was able to significantly repress the expression of GFP only when miR-24a sites in the 3'UTR of caspase9 or apaf1 were present (Fig. 5B). This demonstrates that $m i R-24 a$ can specifically target the $m i R-24 a$ binding sites in the 3'UTRs of both caspase9 and apaf1. The caspase9 3'UTR may have more miR-24a-binding sites than the two we identified, as the mutated version is still slightly repressed relative to the "no UTR" control.

\section{miR-24a is able to prevent hydroxyurea-induced apoptosis}

Our loss-of-function studies determined that $m i R-24 a$ is necessary in the eye to prevent apoptosis from occurring, 
A

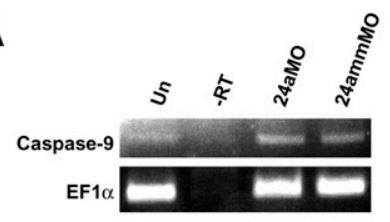

B
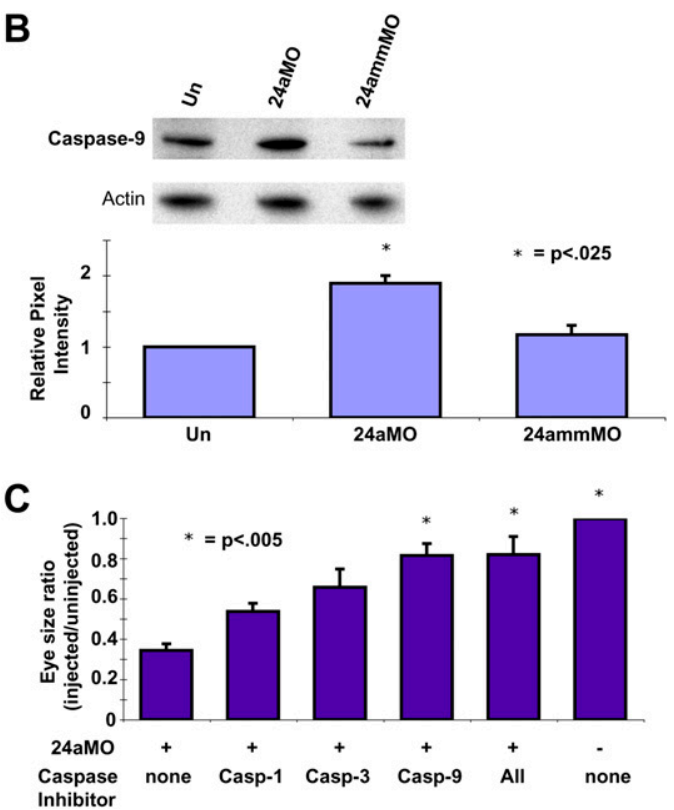

Figure 4. Knockdown of $m i R-24 a$ causes up-regulation of Caspase9 protein and is rescuable by its inhibition. (A) RT-PCR of caspase 9 on stage 28 heads shows no change in mRNA levels between uninjected and miR-24a knockdown embryos. (B) Western blot for Caspase9 on stage 28 heads shows a significant increase in protein levels when $m i R-24 a$ function is blocked ( $n=3$ experiments; error bars are SEM). (C) Coinjection of Caspase inhibitors to rescue the miR-24a knockdown phenotype. Only a pan-Caspase inhibitor (All) or an inhibitor specific to Caspase9 rescued the reduction in eye size caused by injection of $24 \mathrm{aMO}$ ( $n \geq 47$ for each treatment; error bars are SEM).

and that depletion of miR-24a leads to the development of a smaller eye. To test whether miR-24a is sufficient to regulate other modes of apoptosis, we induced apoptosis by soaking embryos in $15 \mathrm{mM}$ hydroxyurea; this causes large amounts of Caspase-dependent apoptosis, visible as cell autolysis and loss of adhesion, but only at the onset of zygotic transcription (Stack and Newport 1997; Takayama et al. 2004). To assess miR-24a-mediated rescue, we injected $2 \mathrm{ng}$ of $\mathrm{miR}-24 a$ into both cells of a two-cell stage embryo and incubated them in $15 \mathrm{mM}$ hydroxyurea until early gastrula stages, then analyzed the number of embryos undergoing apoptosis. We found that the addition of $m i R-24 a$ significantly reduced the number of embryos undergoing apoptosis, and that this reduction was not seen when embryos were injected with equal amounts of a 4-base-pair mismatch RNA (miR-24amm) (Fig. 5C). These results indicate that miR-24a is able to prevent apoptosis more generally than in the eye, most likely by targeting components of the apoptosis pathway including caspase 9 and apaf1.

\section{Conclusions}

We found that $m i R-24 a$ represses apoptosis in the neural retina, likely by down-regulating apaf1 and caspase9.
These two proteins are the major components of the apoptosome, the first complex in the protease cascade deployed during programmed cell death. Normally, Cytochrome $c$ released from the mitochondria binds Apaf1 and induces the recruitment of Caspase9 to the apoptosome. The interaction between these proteins leads to the activation of Caspase9, which then cleaves and activates downstream Caspases, including Caspase3, Caspase6, and Caspase7 (Guerin et al. 2006). Our data implicate miRNAs as important regulators of the apoptosome during vertebrate development. Other genes also may be regulated by miR-24a in this process and make a contribution to the knockdown phenotype. It is intriguing that $m i R-23 b$ and $m i R-27 b$, the other miRNAs in the cluster with $m i R-24 a$, are predicted to regulate other genes important for apoptosis, such as the lowaffinity NGF receptor or diablo, an antagonist of inhibitor
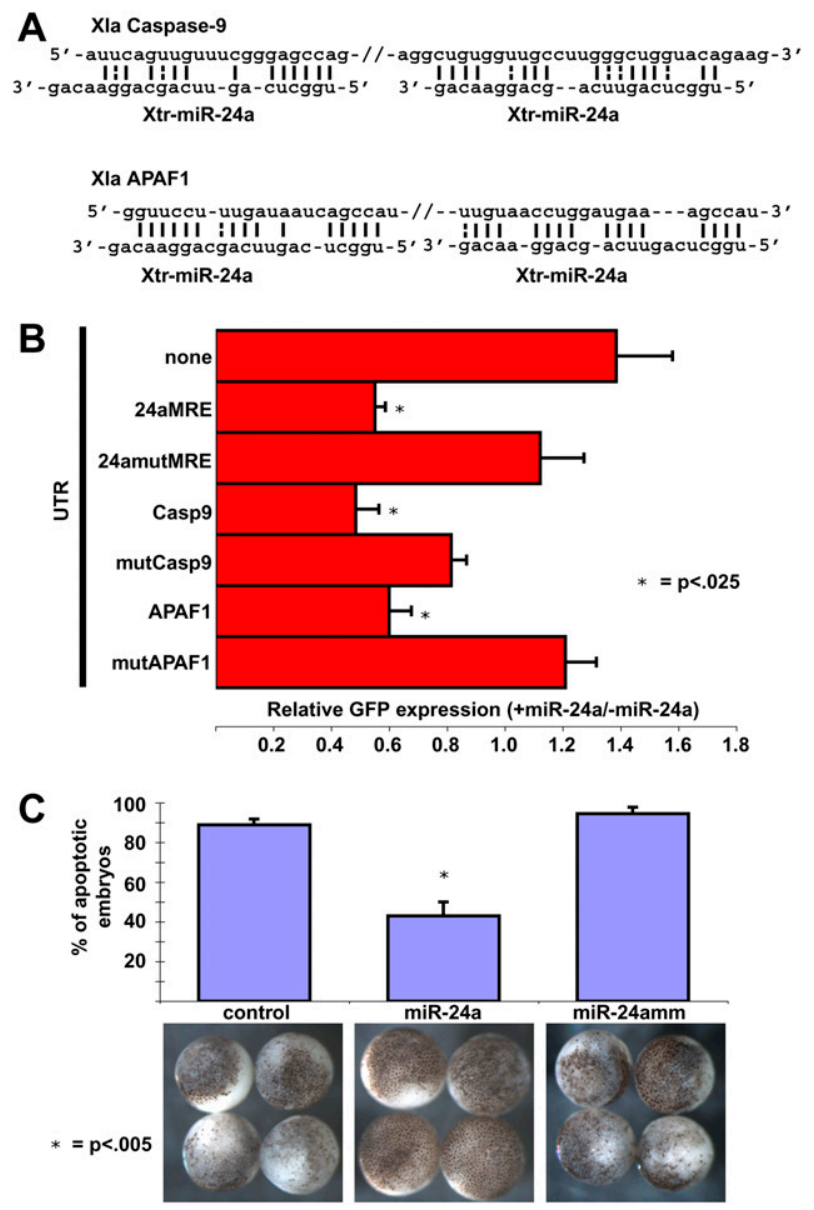

Figure 5. miR-24a targets the 3' UTR of caspases and apaf1. (A) The 3'UTRs of $X$. laevis caspase 9 and apaf1 have two putative miR24a-binding sites, as measured by RNAhybrid (Kruger and Rehmsmeier 2006). (B) The addition of $m i R-24 a$ causes GFP reporters with miR-24a recognition elements in their $3^{\prime} \mathrm{UTRs}$ to be repressed. GFP reporters with the 3'UTR of caspase 9 and apaf1 show repression by $m i R-24 a$. Mutating the putative miR-24a-binding sites in these constructs (mutCasp9 and mutApaf1) abolishes the repression $(n=4$ experiments; error bars are SEM). $(C)$ Embryos injected with $2 \mathrm{ng}$ of $m i R-24 a$ or $m i R-24 a m m$ or nothing (control) were cultured in $15 \mathrm{mM}$ hydroxyurea to induce apoptosis at the onset of gastrulation. Only $m i R-24 a$ was able to significantly repress or delay the onset of apoptosis ( $n=3$ experiments; error bars are SEM). 
of apoptosis proteins (IAPs) (Guerin et al. 2006; GriffithsJones et al. 2008).

This study also makes clear the importance of proper regulation of apoptosis in the neural retina in $X$. laevis. Mouse knockouts of caspase 9 have an excessive accumulation of neurons throughout the CNS, including the retina (Hakem et al. 1998). Knockouts of apaf1 also have retinal overgrowth in addition to forebrain overgrowth and thickening of the hindbrain walls (Cecconi et al. 1998). Here, we show that disrupting a negative regulator of these genes has the opposite effect to their elimination, resulting in higher levels of apoptosis and a smaller eye. Previous reports have disrupted the transcription of apaf1 by interfering with histone deacetylation, causing an increase in apoptosis in the developing retina (Wallace and Cotter 2008). Our data argue that post-transcriptional regulation by miRNAs also may be a factor in the correct regulation of apaf1 during normal amphibian development.

Mouse knockouts of caspase 3 can have a similar phenotype to those of caspase9, though the phenotype occurs in far fewer animals (10\% vs. $97 \%)$, and cell death is still seen in the retina (Cecconi et al. 2008). This agrees with our experiments using Caspase inhibitors: While the Caspase3 inhibitor was able to partially rescue the phenotype caused by depletion of $m i R-24 a$, the rescue was not significant, indicating that it probably has only a small role in the downstream activity of the Caspase cascade.

Remarkably, the loss of a single miRNA is sufficient to release members of the apoptotic pathway from inhibition, resulting in a significant increase in apoptosis and severe morphological disruption of eye structures. This occurs without any disruption in early patterning or specification. This indicates that $m i R-24 a$ functions primarily as a negative regulator of apoptosis in the neural retina. Similar miRNA regulation may be important for other neural structures that also require precise levels of apoptosis during development, including the brain and spinal cord (De Pietri Tonelli et al. 2008). Further characterization of the functions of neuronally expressed miRNAs should lead to an understanding of how general this mechanism is during development.

\section{Materials and methods}

pri-miRNA in situ hybridization and $R T-P C R$

$X$. tropicalis in situ hybridizations for primary miRNA transcripts were done as in Walker and Harland (2008) and RT-PCRs were performed as in Sive et al. (2000). The primers used were as folows: for pri-miR-24a, 5'-GGACCTGTCCTCTTGTGCC-3' and 5'-GCTCAAGGGCCCGACTC-3'; for otx2, 5'-CGGGATG GATTTGTTGCA-3' and 5'-TTGAACCAGACCTGGACT-3'; for ornithine decarboxylase, 5'-GTCAATGATGGAGTGTAT-3' and $5^{\prime}$-TTCCATTCCGCTCTCCTGAG-3'; for caspase 9 , 5' -CAG AATGCGCTCCTTTCACTTTG-3' and $5^{\prime}$-AAGTAGCTCACG ATTCTCTCTAC-3'; and for $e f 1 \alpha$ as in Krieg et al. (1989).

\section{4aMO injections and eye size measurements}

$X$. laevis eggs were collected and fertilized and embryos were cultured by standard procedures (Sive et al. 2000). An antisense oligonucleotide morpholino designed to block miR-24a was ordered from GeneTools, LLC, with the sequence 5'CTGTTCCTGCTGAACTGAGCCAGTG-3' (24aMO), as well as a mismatched morpholino with the sequence $5^{\prime}$-CTGTTC
CTGCTGAACACAGGGAGTG-3' (24ammMO). The dose injected throughout our analyses was $20 \mathrm{ng}$ in one cell at the twocell stage, except where noted otherwise.

Eye size was quantified by measuring the anterior-posterior diameter of the eye at its greatest point at stage 40 (staging according to Nieuwkoop and Faber [1994]) using a Zeiss Stemi SV6 dissecting scope with an eyepiece reticle.

\section{Western blots}

Embryos were injected and cultured until stage 28. Heads were dissected away from bodies and prepared for Westerns as in Sive et al. (2000). Actin antibody (Santa Cruz Biotechnologies, \#H3105) was used at 1:4000 and Caspase9 antibody (Abcam, ab25758) was used at 1:200 overnight. A secondary antibody (Jackson Immunoresearch, HRP-conjugated donkey anti-rabbit IgG) was used at 1:5000. Actin ran at $\sim 45 \mathrm{kDa}$ and full-length Caspase 9 at ran $\sim 47 \mathrm{kDa}$.

\section{Fluorescent protein injection and quantification}

Katushka RFP RNA was coinjected with every GFP experiment at 200 pg per cell. All RNA for injection was made using SP6 RNA polymerase mMessage mMachine kit (Ambion) and AscIdigested template. RNA for GFP constructs was injected at a dose of $1 \mathrm{ng}$ per cell into both cells of the two-cell stage embryo. MiR$24 a$ and $m i R-133 b$ RNA mimicking the endogenous hairpin (Integrated DNA Technologies) were miR-24aTop (5'-GUGCC UACUGAACUGAUAUCAGU-3'), miR-24aBot (5'-UGGCUCA GUUCAGCAGGAACAG-3'), miR-133bTop (5'-UUGGUCCCC UUCAACCAGCUA-3'), and $m i R-133 b$ Bot (5'-GCUGGUCAAA CGGAACCAAUU-3'). RNAs were combined and diluted to a stock concentration of $1 \mu \mathrm{g} / \mu \mathrm{L}$, heated for $1 \mathrm{~min}$ to $80^{\circ} \mathrm{C}$, and then allowed to cool to room temperature to form duplexes. The same was done for $m i R-24 m m$ Top (5'-GUGGGUACUGAA CUGAUAAGAGU-3') and miR24mmBot (5'-UCUCUCAGUU CAGCACCAACAG-3'). All photos from a particular experiment were identically batch-processed using Photoshop (Adobe), and ImageJ1.34S (Wayne Rasband, NIH, Bethesda, MD) was used to measure average pixel intensity of RFP and GFP. The ratio between GFP and RFP was then used for comparison. All statistical significance tests were done using the unpaired Student t-test.

\section{Immunohistochemistry and TUNEL}

Cryosections were as in Zaghloul and Moody (2007). Phosphorylated histone3 (Upstate Biotechnologies) staining was done as in Ruan et al. (2006). TUNEL staining was performed as in Hensey and Gautier (1997), using BM Purple for the staining (Roche).

\section{Caspase inhibitors}

Caspase inhibitors (CalBiochem) were caspase-1 inhibitor I (AcYVAD-CHO), caspase-3 inhibitor II (Z-DEVD-FMK), caspase inhibitor I (Z-VAD-FMK), and caspase-9 inhibitor III (Ac-LEHD$\mathrm{CMK})$, all used at $1 \mathrm{ng}$ per injection. Stocks were diluted in DMSO or distilled water.

\section{Hydroxyurea treatment}

Hydroxyurea (Sigma) was diluted in $1 / 3 \times$ MR solution to a final concentration of $15 \mathrm{mM}$ and embryos were treated from $2 \mathrm{~h}$ post-fertilization until stage 10.5, then fixed in MEMFA (Sive et al. 2000). 


\section{Acknowledgments}

We thank S. Amacher for the contribution of the original destabilized GFP plasmid, and members of the Harland laboratory for valuable discussion and advice. R.M.H. is supported by grants from the NIH (GM 42341).

\section{References}

Cecconi, F., Alvarez-Bolado, G., Meyer, B.I., Roth, K.A., and Gruss, P. 1998. Apaf1 (CED-4 homolog) regulates programmed cell death in mammalian development. Cell 94: 727-737.

Cecconi, F., Piacentini, M., and Fimia, G.M. 2008. The involvement of cell death and survival in neural tube defects: A distinct role for apoptosis and autophagy? Cell Death Differ. 15: 1170-1177.

Chan, J.A., Krichevsky, A.M., and Kosik, K.S. 2005. MicroRNA-21 is an antiapoptotic factor in human glioblastoma cells. Cancer Res. 65: 6029-6033.

Cheng, A.M., Byrom, M.W., Shelton, J., and Ford, L.P. 2005. Antisense inhibition of human miRNAs and indications for an involvement of miRNA in cell growth and apoptosis. Nucleic Acids Res. 33: 12901297.

Damiani, D., Alexander, J.J., O'Rourke, J.R., McManus, M., Jadhav, A.P., Cepko, C.L., Hauswirth, W.W., Harfe, B.D., and Strettoi, E. 2008. Dicer inactivation leads to progressive functional and structural degeneration of the mouse retina. J. Neurosci. 28: 4878-4887.

de la Rosa, E.J. and de Pablo, F. 2000. Cell death in early neural development: Beyond the neurotrophic theory. Trends Neurosci. 23: 454-458.

De Pietri Tonelli, D., Pulvers, J.N., Haffner, C., Murchison, E.P., Hannon, G.J., and Huttner, W.B. 2008. miRNAs are essential for survival and differentiation of newborn neurons but not for expansion of neural progenitors during early neurogenesis in the mouse embryonic neocortex. Development 135: 3911-3921.

Gaze, R.M. and Grant, P. 1992. Spatio-temporal patterns of retinal ganglion cell death during Xenopus development. J. Comp. Neurol. 315: 264-274.

Griffiths-Jones, S., Saini, H.K., van Dongen, S., and Enright, A.J. 2008. miRBase: Tools for microRNA genomics. Nucleic Acids Res. 36: D154-D158. doi: 10.1093/nar/gkm952.

Guerin, M.B., McKernan, D.P., O'Brien, C.J., and Cotter, T.G. 2006. Retinal ganglion cells: Dying to survive. Int. J. Dev. Biol. 50: 665-674.

Hakem, R., Hakem, A., Duncan, G.S., Henderson, J.T., Woo, M., Soengas, M.S., Elia, A., de la Pompa, J.L., Kagi, D., Khoo, W., et al. 1998. Differential requirement for caspase 9 in apoptotic pathways in vivo. Cell 94: 339-352.

He, L., He, X., Lim, L.P., de Stanchina, E., Xuan, Z., Liang, Y., Xue, W., Zender, L., Magnus, J., Ridzon, D., et al. 2007. A microRNA component of the p53 tumour suppressor network. Nature 447: 1130-1134.

Hensey, C. and Gautier, J. 1997. A developmental timer that regulates apoptosis at the onset of gastrulation. Mech. Dev. 69: 183-195.

Krieg, P.A., Varnum, S.M., Wormington, W.M., and Melton, D.A. 1989. The mRNA encoding elongation factor $1-\alpha(E F-1 \alpha)$ is a major transcript at the midblastula transition in Xenopus. Dev. Biol. 133: 93-100.

Kruger, J. and Rehmsmeier, M. 2006. RNAhybrid: microRNA target prediction easy, fast and flexible. Nucleic Acids Res. 34: W451-W454. doi: $10.1093 / \mathrm{nar} / \mathrm{gk} 1243$.

Makeyev, E.V., Zhang, J., Carrasco, M.A., and Maniatis, T. 2007. The microRNA miR-124 promotes neuronal differentiation by triggering brain-specific alternative pre-mRNA splicing. Mol. Cell 27: 435-448.

Mayordomo, R., Valenciano, A.I., de la Rosa, E.J., and Hallbook, F. 2003. Generation of retinal ganglion cells is modulated by caspasedependent programmed cell death. Eur. J. Neurosci. 18: 1744-1750.

Nieuwkoop, P.D. and Faber, J. 1994. Normal table of Xenopus laevis (Daudin): A systematical and chronological survey of the development from the fertilized egg till the end of metamorphosis. Garland, New York.

O'Driscoll, C., Donovan, M., and Cotter, T.G. 2006. Analysis of apoptotic and survival mediators in the early post-natal and mature retina. Exp. Eye Res. 83: 1482-1492.
Pasquinelli, A.E., Hunter, S., and Bracht, J. 2005. MicroRNAs: A developing story. Curr. Opin. Genet. Dev. 15: 200-205.

Penaloza, C., Orlanski, S., Ye, Y., Entezari-Zaher, T., Javdan, M., and Zakeri, Z. 2008. Cell death in mammalian development. Curr. Pharm. Des. 14: 184-196.

Ruan, G., Wedlich, D., and Koehler, A. 2006. Xenopus cadherin-6 regulates growth and epithelial development of the retina. Mech. Dev. 123: 881-892.

Saka, Y. and Smith, J.C. 2001. Spatial and temporal patterns of cell division during early Xenopus embryogenesis. Dev. Biol. 229: $307-$ 318.

Sive, H.L., Grainger, R.M., and Harland, R.M. 2000. Early development of Xenopus laevis: A laboratory manual. Cold Spring Harbor Laboratory Press, Cold Spring Harbor, NY.

Stack, J.H. and Newport, J.W. 1997. Developmentally regulated activation of apoptosis early in Xenopus gastrulation results in cyclin A degradation during interphase of the cell cycle. Development 124: 3185-3195.

Takayama, E., Higo, T., Kai, M., Fukasawa, M., Nakajima, K., Hara, H., Tadakuma, T., Igarashi, K., Yaoita, Y., and Shiokawa, K. 2004. Involvement of caspase- 9 in execution of the maternal program of apoptosis in Xenopus late blastulae overexpressed with S-adenosylmethionine decarboxylase. Biochem. Biophys. Res. Commun. 325: 1367-1375.

Vecino, E., Hernandez, M., and Garcia, M. 2004. Cell death in the developing vertebrate retina. Int. J. Dev. Biol. 48: 965-974.

Walker, J.C. and Harland, R.M. 2008. Expression of microRNAs during embryonic development of Xenopus tropicalis. Gene Expr. Patterns 8: 452-456.

Wallace, D.M. and Cotter, T.G. 2008. Histone deacetylase activity in conjunction with E2F-1 and p53 regulates Apaf-1 expression in $661 \mathrm{~W}$ cells and the retina. J. Neurosci. Res. 87: 887-905.

Wallace, D.M., Donovan, M., and Cotter, T.G. 2006. Histone deacetylase activity regulates apaf- 1 and caspase 3 expression in the developing mouse retina. Invest. Ophthalmol. Vis. Sci. 47: 2765-2772.

$\mathrm{Xu}, \mathrm{P}$. , Guo, M., and Hay, B.A. 2004. MicroRNAs and the regulation of cell death. Trends Genet. 20: 617-624.

Yin, V.P., Thomson, J.M., Thummel, R., Hyde, D.R., Hammond, S.M., and Poss, K.D. 2008. Fgf-dependent depletion of microRNA-133 promotes appendage regeneration in zebrafish. Genes \& Dev. 22: 728-733.

Zaghloul, N.A. and Moody, S.A. 2007. Alterations of $\mathrm{rx1}$ and pax6 expression levels at neural plate stages differentially affect the production of retinal cell types and maintenance of retinal stem cell qualities. Dev. Biol. 306: 222-240.

Zhao, Y., Samal, E., and Srivastava, D. 2005. Serum response factor regulates a muscle-specific microRNA that targets Hand2 during cardiogenesis. Nature 436: 214-220. 


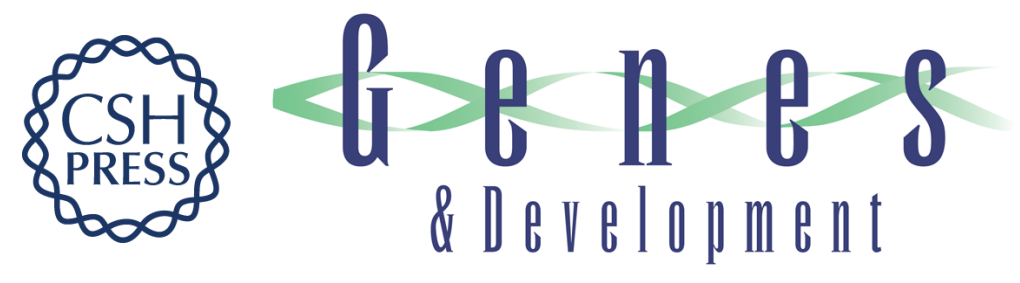

\section{microRNA-24a is required to repress apoptosis in the developing neural retina}

James C. Walker and Richard M. Harland

Genes Dev. 2009, 23: originally published online April 16, 2009

Access the most recent version at doi:10.1101/gad.1777709

\section{Supplemental http://genesdev.cshlp.org/content/suppl/2009/04/29/gad.1777709.DC1 Material}

References This article cites 32 articles, 6 of which can be accessed free at: http://genesdev.cshlp.org/content/23/9/1046.full.html\#ref-list-1

\section{License}

Email Alerting

Receive free email alerts when new articles cite this article - sign up in the box at the top Service

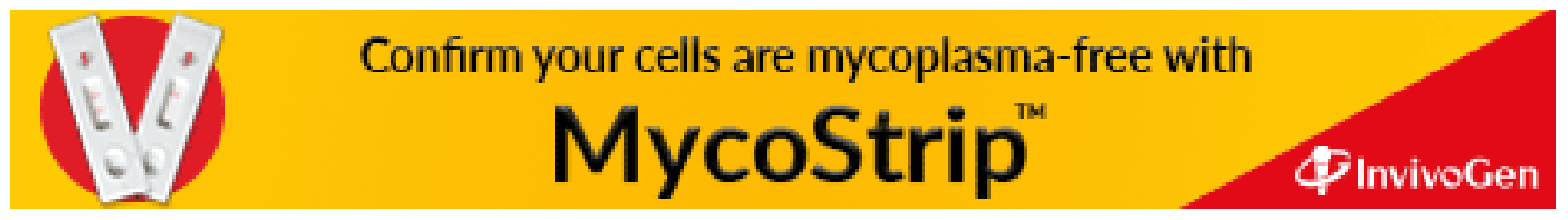

